\title{
Evaluación de tierras para el cultivo de caña de azúcar (Saccharum officinarum) en el noroeste de la provincia de Corrientes, Argentina
}

\author{
Perucca, Sandra C.; Kurtz, Ditmar B. \\ INTA EEA Corrientes. Ruta 12 km 1.008 E-mail: perucca.sandra@inta.gob.ar
}

\begin{abstract}
RESUMEN
La evaluación de tierras clasifica los suelos en categorías de aptitud fácilmente entendible para el público en general, lo que la hace particularmente útil para planificar el uso del territorio. En este trabajo se evaluó la aptitud de las tierras para el cultivo de caña de azúcar en el noroeste de la provincia de Corrientes aplicando el esquema FAO, considerando dos niveles tecnológicos contrastantes y utilizando la información básica de suelos existente, en dos escalas de detalle disponibles (1:250.000 y 1:50.000). Los resultados de la evaluación se presentan mediante tablas comparativas con la taxonomía de los suelos, la capacidad de uso y el índice de productividad. Se utilizó el software QGIS2.8.2 para la representación cartográfica. Casi un cuarto del área total evaluada posee suelos aptos para el cultivo de caña de azúcar con bajo nivel de adopción de tecnología. Mientras que, aplicando el paquete tecnológico completo, aproximadamente un $8 \%$ de los suelos pasarían a ser muy aptos. El sector norte, donde se efectuó la evaluación a escala más detallada, casi el 30\% de la superficie posee tierras aptas para el cultivo, de las cuales el 10\% son muy aptas si se aplica un manejo mejorado.
\end{abstract}

Palabras clave: aptitud, cartografía, modelos, suelos.

\begin{abstract}
SUMMARY
Land evaluation classified soils according its use aptitude and provide a tool for land use planning. At this study soil aptitude for sugar cane production was evaluated at Corrientes Northwest using FAO scheme, considering two technological levels (low and high) and two scale soil map (1:250000; 1:50000). Data is presented through soil taxonomy comparative tables, use capacity and productivity index. Software QGIS2.8.2 was used for cartographic maps. Almost a quarter of total evaluated area had suitable soils for sugar cane crop using low technology level. Using a complete technology package, $8 \%$ of soils could become suitable for that crop. The North part, where evaluation was made using more detailed scale map, showed that $30 \%$ area had suitable soils for this crop. Using a higher technological level production, a $10 \%$ of this area could be improved.
\end{abstract}

Key words: land suitability, cartography modeling, soils.

\section{INTRODUCCIÓN}

El aprovechamiento de las potencialidades ambientales y productivas de un territorio requiere de información precisa para la toma de decisiones. La evaluación de tierras es muy útil en la planificación del uso del territorio ya que permite "traducir" la información básica y taxonómica de los suelos, que a veces no es sencilla de interpretar, en categorías de aptitud fácilmente entendibles, incluso, para expertos de otras disciplinas no relacionadas a la edafología. Existen numerosos métodos de evaluación para predecir la capacidad de los suelos para soportar un uso específico. En Corrientes, los suelos se han evaluado básicamente de acuerdo a su capacidad de uso (CU) o asignándole valores numéricos a través del índice de productividad (IP) (Escobar et al., 1996). Más recientemente las evaluaciones para fines

Recibido:04/08/2015. Aceptado:21/04/2016 específicos, a través del esquema FAO, han adquirido más importancia, básicamente por dar respuesta a usos potenciales o actuales específicos y no generales (Ligier et al., 2001, 2012 y 2014).

El cultivo de caña de azúcar (Saccharum officinarum) constituye una alternativa viable para la ganadería de pequeña y mediana escala que caracteriza a la región y las condiciones agroclimáticas locales, si bien no se encuentran libres de limitaciones, resultan adecuadas para el normal crecimiento del cultivo (Kurtz y Barrios, 2014).

Partiendo de información básica de suelos a una escala 1:250.000 (Matteio, inédito), se realizó el análisis de aptitud específica para caña de azúcar en la región noroeste de la Provincia de Corrientes correspondiente a los departamentos Empedrado, Capital, San Cosme, San Luis del Palmar e Itatí, abarcando aproximadamente 660.000 hectáreas (Figura 1a). Además, se efectuó el mismo análisis a mayor de- 

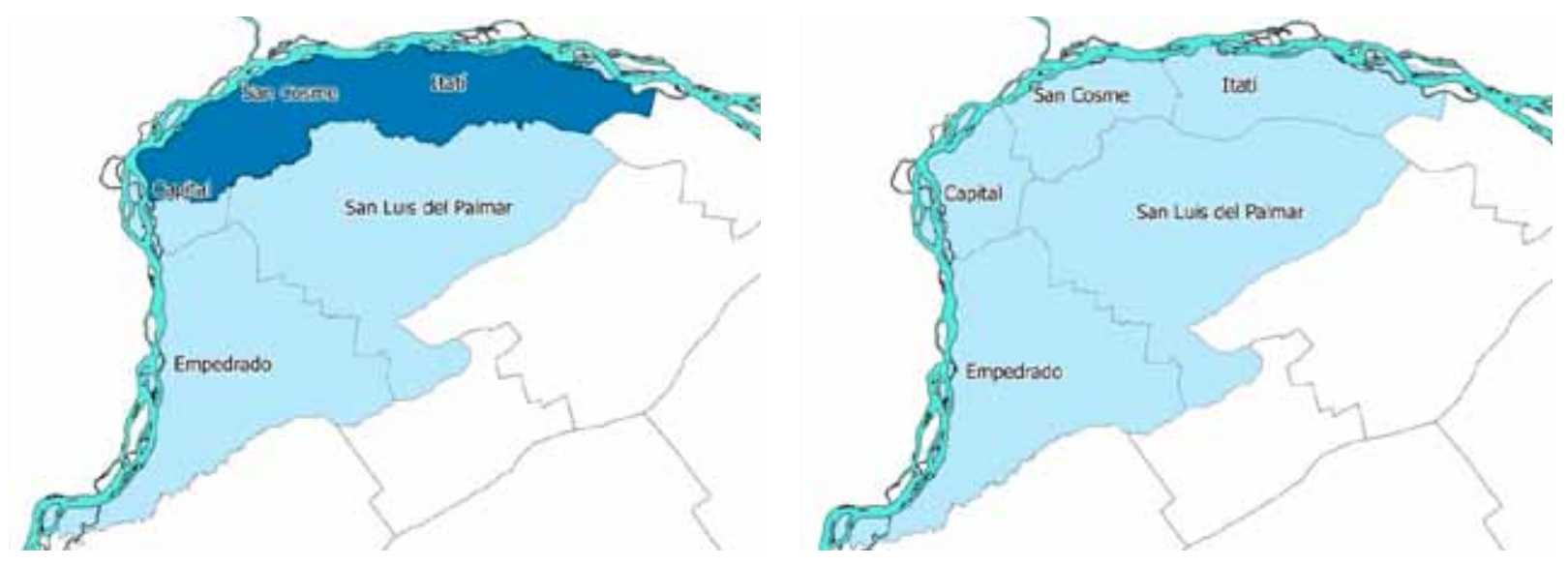

Figura 1. Áreas evaluadas: a) Territorio Noroeste de Corrientes: departamentos Empedrado, Capital, San Cosme, San Luis del Palmar e Itatí. Escala 1:250.000. b) Norte del Territorio Noroeste de Corrientes: departamentos de Itatí, San Cosme y parte del departamento Capital. Escala 1:50.000.

talle (1:50.000) (Escobar et al., 1983) en un área de 168.500 hectáreas, ubicadas en el sector norte del territorio provincial (Figura 1b).

\section{OBJETIVO}

Evaluar la aptitud de las tierras para el cultivo de caña de azúcar en la región noroeste de la Provincia de Corrientes.

\section{MATERIALES Y MÉTODOS}

La evaluación de la aptitud de tierras para fines específicos se realizó empleando el marco conceptual de la FAO (FAO, 2003), utilizando el sistema automatizado de evaluación de tierras (Automated Land Evaluation System - ALES) (Rossiter, 1990; Rossiter. 1994; y Rossiter et al., 1995). Este método se basa en confrontar los requerimientos del cultivo (demanda) bajo un nivel de manejo predeterminado, con la información generada en los levantamientos de suelos (oferta). La evaluación implica las siguientes etapas: Definición de los tipos de utilización de la tierra (TUT); Definición de los requisitos de uso de la tierra (RUT); Conceptualización de los árboles de decisión para cada TUT; Ingreso al ALES de características

Tabla 1. Caracterización de los tipos de uso de la tierra para caña de azúcar.

\begin{tabular}{lll}
\hline & TUT1- Básico & TUT2 - Mejorado \\
\hline Superficie & $<3$ ha & $>3 \mathrm{ha}$ \\
Rendimiento & $<50 \mathrm{Mg} \mathrm{ha}^{-1}$ & $>50 \mathrm{Mg} \mathrm{ha}^{-1}$ \\
Destino & Alimentación animal & Valor agregado (carne, leche, queso) \\
Mano de obra & Familiar & Familiar y/o externa \\
Tracción & Animal & Mecánica \\
Maquinaria & No & Propia y/o contratada \\
Asociación & Si/no & Sí \\
Origen caña semilla & De la zona, desconocido & Conocido \\
Herbicidas & No & Sí \\
Fertilización & No & Sí (de arranque y todos los años) \\
Densidad de plantación & 1,5 a 3 m entre lineos simples & 1,4 a 1,6 m entre lineos apareados a 0,4 m \\
Cosecha & Manual & Manual o mecánica \\
\hline
\end{tabular}

Tabla 2. Características y cualidades utilizadas para definir las Clases de aptitud de las tierras.

\begin{tabular}{llll}
\hline $\begin{array}{l}\text { Condiciones } \\
\text { para la plantación }\end{array}$ & $\begin{array}{l}\text { Condiciones } \\
\text { para el desarrollo }\end{array}$ & $\begin{array}{l}\text { Condiciones } \\
\text { de fertilidad }\end{array}$ & $\begin{array}{l}\text { Condiciones } \\
\text { para la cosecha }\end{array}$ \\
\hline Textura del horizonte superficial & $\begin{array}{l}\text { Profundidad efectiva } \\
\text { Espesor del horizonte A }\end{array}$ & IFG** & Profundidad de la napa estacional \\
$\begin{array}{l}\text { Drenaje*** } \\
\text { Subpaisaje }\end{array}$ & $\begin{array}{l}\text { Riesgo de erosión } \\
\text { Drenaje*** }\end{array}$ & \\
\hline
\end{tabular}

$\left(^{*}\right)$ PSI: Porcentaje de sodio intercambiable; $(* *)$ Índice de fertilidad global (IFG). (***) El drenaje es una cualidad de peso en ambas etapas del cultivo. 
Tabla 3. Series de suelos clasificadas por aptitud específica para caña de azúcar. Escala 1:250.000.

\begin{tabular}{|c|c|c|c|c|c|}
\hline Serie & Clasificación taxonómica & CU & IP & TUT1 & TUT2 \\
\hline Andreau & Epiacualfes típicos & Vw & 15 & NA & NA \\
\hline Arroyo Riachuelo & Calciacuoles típicos & VIw & 6 & NA & NA \\
\hline Cañada Burgo & Argiacuoles abrúpticos & VIw & 9 & NA & NA \\
\hline Cañada Carí & Albacualfes vérticos & Vw & 12 & NA & NA \\
\hline Carrizal & Endoacueptes mólicos & VIIw & 5 & NA & NA \\
\hline Chavarría & Psamacuentes típicos & Vws & 16 & Marg & Marg \\
\hline Chavarría fpp* (ex Pampín) & Psamacuentes típicos & IVsw & 20 & Mod & Mod \\
\hline Chequín & Albacualfes típicos & Vw & 9 & NA & NA \\
\hline Costa Grande & Albacualfes típicos & VIw & 7 & NA & NA \\
\hline Ensenada Grande & Udipsamentes árgicos & Illes & 33 & Mod & MA \\
\hline Ipucú & Natracualfes típicos & VIs & 2 & NA & NA \\
\hline La Ángela & Hapludalfes arénicos & IVs & 28 & Mod & Mod \\
\hline Lomas de Empedrado & Paleudalfes ródicos & Illes & 33 & Mod & MA \\
\hline Mandiyurá & Glosacualfes típicos & VIw & 5 & NA & NA \\
\hline Obregón & Argialboles argiácuicos & VIw & 10 & Marg & Marg \\
\hline Ocá & Natracualfes vérticos & VIIs & 2 & NA & NA \\
\hline Parada Coco & Paleudoles típicos & Ile & 52 & Mod & $\mathrm{MA}$ \\
\hline Paso Patria & Albacualfes vérticos & Vw & 6 & NA & NA \\
\hline Pexoa & Udipsamentes ácuicos & Vs & 16 & Mod & Mod \\
\hline Puerto Corazón & Argiudoles vérticos & Ile & 66 & Mod & MA \\
\hline Riacho Grande & Fluvacuentes aéricos & VIIw & 8 & NA & NA \\
\hline Rincón de Ambrosio & Paleudoles típicos & Ile & 50 & Mod & MA \\
\hline Rincón del Madregón & Glosacualfes típicos & VIw & 7 & NA & NA \\
\hline Scorza Cué & Paleudoles ácuicos & Ills & 42 & Mod & MA \\
\hline Tala & Epiacualfes típicos & Vw & 15 & Marg & Marg \\
\hline Tataré & Endoacualfes típicos & Vw & 7 & NA & NA \\
\hline Timbó Paso & Natracuoles típicos & Vls & 7 & NA & NA \\
\hline Toro Isla & Halacueptes aéricos & VIIs & 1 & NA & NA \\
\hline Treviño & Argiudoles ácuicos & Ille & 50 & Mod & Mod \\
\hline Valencia & Endoacualfes típicos & VIw & 5 & NA & NA \\
\hline
\end{tabular}

(*) fpp: fase por profundidad.

Tabla 4. Síntesis de superficies de suelos agrupadas y clasificadas por aptitud específica para cada TUT. Escala 1:250.000.

\begin{tabular}{lcc}
\hline Aptitud específica y Superficie & TUT1 (ha) & TUT2 (ha) \\
\hline Muy Apta & - & 40.855 \\
Moderadamente Apta & 97.640 & 56.785 \\
Marginalmente Apta & 54.483 & 54.483 \\
No Apta & 508.173 & 508.173 \\
\hline
\end{tabular}

y cualidades seleccionadas; Construcción de los árboles de decisión utilizando ALES; Obtención de la evaluación y clasificación de tierras y Validación de los resultados.

El TUT es la clase de uso de la tierra definida en un grado de detalle mayor que un tipo general de uso, ya que describe las principales prácticas de manejo en un contexto territorial determinado. En este trabajo se evaluaron dos TUT para caña de azúcar: TUT1: Cultivo de caña de azúcar con nivel de manejo básico, TUT2: Cultivo de caña de azúcar con nivel de manejo mejorado. Para la caracterización de los TUT se realizaron consultas a productores y técnicos asesores (Tabla 1). Para la identificación de los RUT se procedió a una revisión bibliográfica sobre el cultivo (Subirós Ruiz, 1995; Da Silva Chagas, 2000; Romero et al., 2009; Vargas Rojas, 2009; Manzatto et al., 2010), determinando características y cualidades relevantes (Tabla 2). En base a los RUT se conceptualizaron y desarrollaron los árboles de decisión, confrontándose la oferta de las tierras con las exigencias del cultivo de caña de azúcar, generándose los resultados de aptitud de las tierras para cada tipo de uso.

El resultado de la evaluación se volcó en un SIG mediante el software libre QGIS2.8.2.

\section{RESULTADOS}

La evaluación a escala 1:250.000 sobre un total de 30 series de suelos (Tablas 3 y 4), arrojó para el TUT1, 10 series con aptitud moderada (Mod) $(97.640 \mathrm{ha}), 3$ con aptitud marginal (Marg) (54.483 ha) y las restantes, 17 series y áreas no agrícolas (508.173 ha), como 


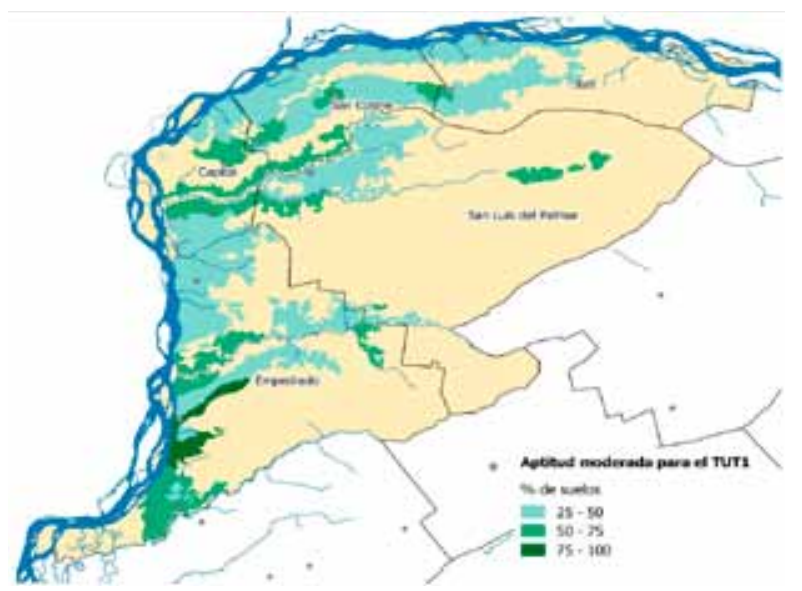

Figura 2. Distribución de los suelos moderadamente aptos para el TUT1 a escala 1:250.000.

no aptas (NA). No se clasificaron suelos en la clase muy apta (MA), principalmente por su baja fertilidad natural.

En el TUT2, con un nivel de manejo mejorado, 6 series pasaron a una categoría superior (de Mod a MA) manifestando su potencial ante un cambio en el nivel de manejo (40.855 ha). Para las demás series no se registraron modificaciones en sus niveles de aptitud; manteniéndose 4 series como Mod (56.785 ha), 3 como Marg (54.483 ha) y finalmente NA (508.173 ha).

Se espera que los rendimientos potenciales definidos para cada clase de aptitud se incrementen al pasar de un tipo de uso a otro, con mayor tecnología. De acuerdo con los resultados obtenidos, existen 97.640 ha (Figura 2) con aptitud moderada para el cultivo de caña de azúcar con un nivel de manejo

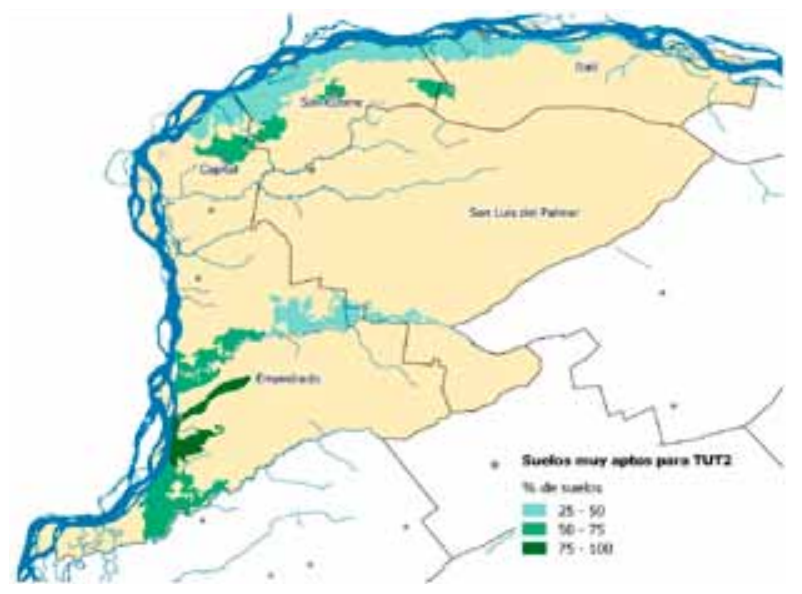

Figura 3. Distribución de los suelos muy aptos para el TUT2 a escala 1:250.000.

básico (TUT1), que al incluir la fertilización anual y buen material genético (TUT2), pasarían 40.855 ha de moderadas a muy aptas (Figura 3).

En cuanto al sector evaluado a escala 1:50.000, sobre un total de 19 series de suelos (Tablas 5 y 6 ), para el TUT1, se registraron 6 series con aptitud Mod (38.844 ha), 2 con aptitud Marg (8.981 ha) mientras que 11 series y áreas no agrícolas (120.675 ha), resultaron NA.

En el TUT2, con nivel de manejo mejorado, 3 de 6 series pasaron a una categoría superior (de Mod a MA) manifestando su potencial ante un cambio positivo en el nivel de manejo (12.017 ha). Para las demás series no se registraron modificaciones en sus niveles de aptitud; manteniéndose 3 series como Mod

Tabla 5. Series de suelos por aptitud específica para caña de azúcar. Escala 1:50.000.

\begin{tabular}{llcccc}
\hline Series & Clasificación & CU & IP & TUT1 & TUT2 \\
\hline Arroyo Riachuelo & Calciacuoles típicos & VIw & 6 & NA & NA \\
Bovadilla & Argialboles típicos & IVs & 15 & Marg & Marg \\
Cañada Mandiyuratí & Albacualfes típicos & VIw & 7 & NA & NA \\
Chavarría & Psamacuentes típicos & Vws & 16 & Marg & Marg \\
Chavarría fpp (ex Pampín) & Psamacuentes típicos & IVsw & 20 & Mod & Mod \\
Chequín & Albacualfes típicos & Vw & 9 & NA & NA \\
Ensenada Grande & Udipsamentes árgicos & Illes & 33 & Mod & MA \\
Indalecio & Natracuoles típicos & VIs & 2 & NA & NA \\
Ipucú & Natracualfes típicos & VIs & 2 & NA & NA \\
Iribú Cuá & Albacualfes udólicos & Vw & 5 & NA & NA \\
Mandiyurá & Glosacualfes típicos & VIw & 5 & NA & NA \\
Ocá & Natracualfes vérticos & VIls & 2 & NA & NA \\
Palmira & Endoacuoles típicos & IVws & 21 & NA & NA \\
Paso Patria & Albacualfes vérticos & Vw & 6 & NA & NA \\
Pexoa & Udipsamentes ácuicos & Vs & 16 & Mod & Mod \\
Puerto Corazón & Argiudoles vérticos & Ile & 66 & Mod & MA \\
Scorza Cué & Paleudoles ácuicos & IIIs & 42 & Mod & MA \\
Toro Isla & Halacueptes aéricos & VIls & 1 & NA & NA \\
Treviño & Argiudoles ácuicos & Ille & 50 & Mod & Mod \\
\hline
\end{tabular}


Tabla 6. Síntesis de superficies de suelos por su aptitud específica para cada TUT. Escala 1:50.000.

\begin{tabular}{lcc}
\hline Aptitud específica & TUT1 (ha) & TUT2 (ha) \\
\hline Muy Apta & - & 12.017 \\
Moderadamente Apta & 38.844 & 26.827 \\
Marginalmente Apta & 8.981 & 8.981 \\
\hline No Apta & 120.675 & 120.675 \\
\hline
\end{tabular}

(26.827 ha), 2 como Marg (8.981 ha) y las restantes NA (120.675 ha).

En las 168.500 ha evaluadas a escala 1:50.000, existen aproximadamente 39.000 ha con aptitud moderada para el cultivo de caña de azúcar con un nivel de manejo básico (TUT1), de las cuales 12.000 ha pasarían a muy aptas con un manejo mejorado (Figura 4 a y b).

\section{DISCUSIÓN DE RESULTADOS}

Existen vastos antecedentes sobre la evaluación de la aptitud general de los suelos en Corrientes (Escobar et al. 1983; 1996; Ligier et al., 2001). Sin embargo, son mucho más escasos los estudios sobre la aptitud específica para caña de azúcar, donde existen cómo únicos antecedentes recientes una evaluación de la aptitud climática para el cultivo de caña de azúcar (Kurtz y Barrios, 2014) y otra evaluación integral realizada sobre 914.300 ha en tierras altas y principalmente arenosas del norte y centro oeste de Corrientes (Ligier et al., 2014).

Cabe aclarar que en la elaboración de los árboles de decisión de aptitud se ha utilizado la cualidad "drenaje", tanto para evaluar las condiciones para la plantación, como para evaluar las condiciones para el desarrollo. Esta cualidad es de suma importancia para la plantación, debido a que combina la rapidez con que se evacúan los excesos de agua para una buena plantación y la disponibilidad de oxígeno, factor sumamente importante para la brotación de la caña semilla (Manzatto et al., 2010; Romero et al., 2009).

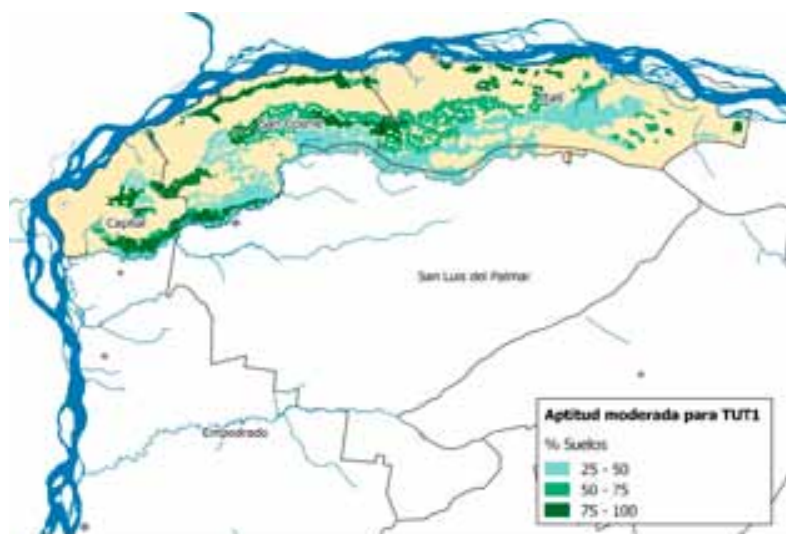

La cualidad drenaje también es determinante para el desarrollo del cultivo y el logro de altos rendimientos para las variedades más difundidas en la zona por las exigencias de drenaje que tiene el cultivo en éste sentido (Manzanato et al., 2010; Romero et al., 2009).

En este estudio presentamos los resultados de una evaluación nunca antes realizada, combinando datos de relevamiento preexistentes (Escobar et al., 1983; 1996; Matteio, inéd.) más la información de campo recabada en un estudio anterior (Ligier et al., 2014) muy cercano a la zona que aquí se ha estudiado.

Trabajos previos en Brasil (Da Silva Chagas, 2000) mencionan que la región sería adecuada para cultivar caña de azúcar, aunque específicamente se ha indicado que en el noroeste de Corrientes no existen zonas totalmente "ideales" libres de limitaciones climáticas, aclarándose que el área estudiada se encontraría dentro del rango normal de crecimiento del cultivo, por el insignificante riesgo de heladas (Kurtz y Barrios 2014).

Si bien la mayor parte de los suelos evaluados no son aptos para caña de azúcar, los resultados indican que existen suelos potencialmente MA cuando el TUT conlleva un manejo mejorado del cultivo, utilizando semilla de calidad e incorporando la fertilización, como ocurre con el TUT2. Vale recordar que para el nivel de aptitud de la clase MA, se suponen rendimientos de entre un $75-100 \%$ del rendimiento potencial esperado para el uso en cuestión (Ligier et al., 2014). Tanto para TUT1 como para TUT2, las clases Mod y Marg presentan las mismas superficies, independientemente de la escala de la información básica para la evaluación. Esto es debido a que las limitaciones de suelos son tales y tan permanentes, que son muy difíciles de corregir mediante el manejo propuesto en TUT2 (Ligier et al., 2014).

\section{CONCLUSIONES}

El análisis efectuado indica que el $23 \%$ de la superficie del sector noroeste podría ser intervenido

Figura 4. Suelos con mejores niveles de aptitud a escala 1:50.000: a) Suelos de aptitud moderada para el TUT1;

b) Suelos muy aptos para el TUT2.

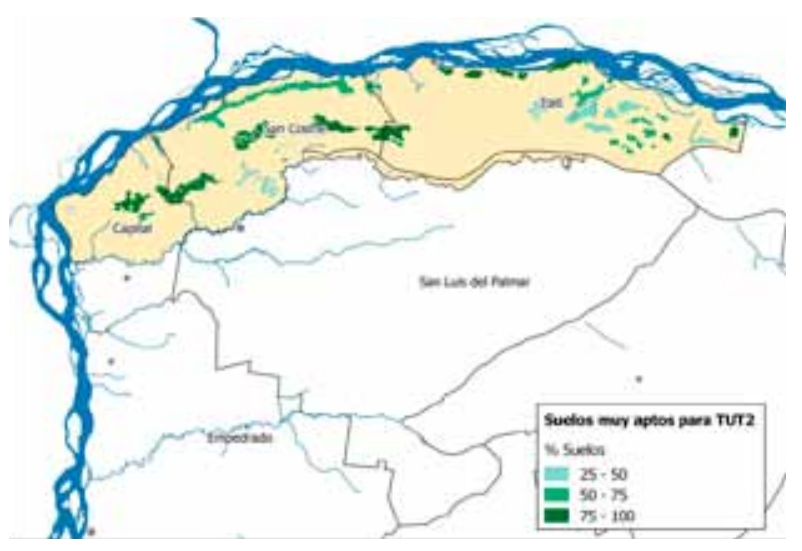


con caña de azúcar. Aplicando fertilización anual y material genético de calidad, unas 40.000 ha tienen el potencial para ser muy aptas, con incrementos de rendimientos esperados de más del $50 \%$.

El sector norte, donde se pudo efectuar la evaluación a escala 1:50.000, arrojó un $28 \%$ de tierras moderada y marginalmente aptas para el cultivo, de las cuales 12.000 ha poseen el potencial de ser muy aptas, aplicando un manejo mejorado.

La información generada con respecto a la aptitud específica de los suelos, representa un aporte al conocimiento del territorio, disponible para los actores involucrados con el mismo.

\section{BIBLIOGRAFIA}

Da Silva Chagas, C. 2000. Zoneamento pedoclimático do Rio Grande do Sul para a cultura da cana-de-açúcar. EMBRAPA. Brasil.

Escobar, E.H., Carnevali, R., Contreras, H.J., Melgar, R., Vallejos, L., Ortigoza, R., Matteio, H.R. y Serra, P. 1983. Los suelos y la vegetación del área de influencia de las obras de Yaciretá. Provincia de Corrientes. INTA. Convenio INTA-CFI-Provincia de Corrientes.

Escobar, E.H.; Ligier, H.D.; Melgar, R; Matteio, H.R; Vallejos, O. 1996. Mapa de suelos de la Provincia de Corrientes. Escala 1:500.000.

FAO. 2003 Evaluación de Tierras con metodologías de FAO: Documento de Trabajo. Proyecto Regional "Ordenamiento Territorial Rural Sostenible" (Proyecto GCP/RLA/139/JPN). Santiago. Chile.

Kurtz, D.B.; Barrios, R. 2014. Aptitud climática para caña de azúcar. Memoria Técnica 2013. INTA EEA Corrientes.

Ligier, H.D.; Kurtz, D.B.; Matteio, H.R.; Perucca, A.R.; Vallejos, O. 2001. Suelos y Vegetación de los departamentos Sauce y Curuzú Cuatiá. Provincia de Corrientes. CFI. Provincia de Corrientes. EEA INTA Corrientes. Recursos Naturales.

Ligier, H.D.; Matteio, H.R.; Garay, J.M.; Vallejos, O.; Estigarribia, C.A.; Kurtz, D.B.; Barrios, R.A.; Ybarra, D.D.; Perucca, A.R.; Sanabria, M.C.; Bernardi, M.J.; Maciel, S.N.; Rey Montoya, T.S.; Matteio, J.P; Flores, C.L. 2012. Mapa de suelos y aptitud de tierras en los Departamentos Esquina, Goya y Lavalle. CFI. Provincia de Corrientes EEA INTA Corrientes. Recursos Naturales

Ligier, H.D.; Matteio, H.R.; Garay, J.M.; Vallejos, O.; Inomata, S.; Kurtz, D.B.; Barrios, R.A.; Ybarra, D.D.; Perucca, A.R.; Perucca, S.C.; Sanabria, M.C.; Bernardi, M.J.; Maciel, S.N.; Rey Montoya, T.S.; Matteio, J.P; Flores, C.L. 2014. Evaluación de tierras para el cultivo de caña de azúcar en la Provincia de Corrientes. CFI Provincia de Corrientes. EEA INTA Corrientes. Recursos Naturales.
Manzatto, C. V.; Baca, J. F. M.; Pereira, S. E. M. 2010 Zoneamento agroecológico da cana-de-açúcar: abordagem metodológica para integração temática de grandes áreas territoriais. En: Prado, R. B.; Turetta, A. P. D.; Andrade, A. G. de (Org.). Manejo e conservação do solo e da agua no contexto das mudanças ambientais. Rio de Janeiro: Embrapa Solos. 486 pp. 193-214.

Matteio, H.R. (Inédito) Digitalización de paisajes y suelos de la Provincia de Corrientes. INTA EEA Corrientes.

Romero, E. R.; Digonzelli, P.A.; Scandaliaris, J. (editores). 2009. Manual del cañero. Primera edición. Las Talitas. Estación Experimental Agroindustrial Obispo Colombres, $232 \mathrm{pp}$.

Rossiter, D.G. 1990. Ales: A Framework for land evaluation using a microcomputer. In Soil Use and Management. Vol $6 \mathrm{~N}^{\circ} 1$.

Rossiter D.G. 1994. Conceptos básicos y procedimientos de evaluación de tierras. Universidad de Cornell. Fac. Agricultura y Ciencias de la Vida. Traducido Proyecto CLAS/ITC. Cochabamba. Bolivia.

Rossiter, D.G.; A. Jiménez T. y Van Wambeke, A. 1995. Sistema Automatizado para la Evaluación de Tierras. ALES. Versión 4.5 en Español. Manual para Usuarios. Cornell University. Ithaca.

Rossiter, D.G.; Vargas Rojas, R. 2004. Metodologías para el levantamiento del recurso suelo. Soil Science Division. ITCc Division Geoinformación suelos y tierras. 2da Versión revisada Marzo 2000. Traducción y adaptación. International Institute for Geo-information Science \& Earth Observation (ITC) 2002.

Subirós Ruiz, F. 1995. El cultivo de la caña de azúcar. pp. 58. Editorial Universidad Estatal a Distancia San José. Costa Rica.

Vargas Rojas, R. 2009. Mapeo digital del suelo y su evaluación con fines de producción de caña de azúcar en los municipios de Ixiamas y San Buenaventura. Conservación Internacional Bolivia y Conservación Estratégica. La Paz, Bolivia. 